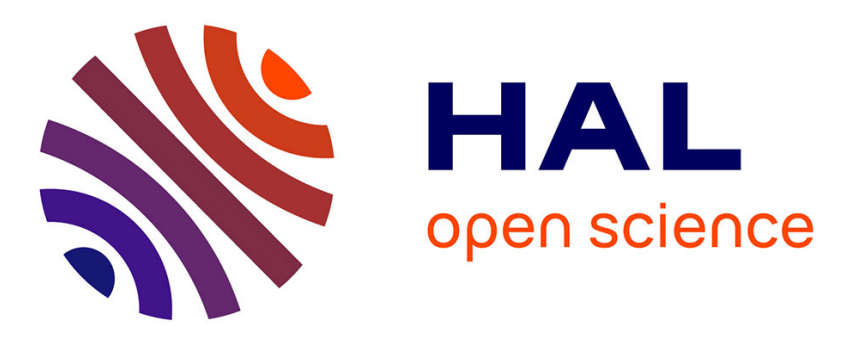

\title{
Weakly multimode dynamics of a photorefractive oscillator
}

Daniel Hennequin, L. Dambly, D. Dangoisse, P. Glorieux

\section{To cite this version:}

Daniel Hennequin, L. Dambly, D. Dangoisse, P. Glorieux. Weakly multimode dynamics of a photorefractive oscillator. Journal de Physique III, 1994, 4 (12), pp.2459-2469. 10.1051/jp3:1994291 . jpa-00249277

\section{HAL Id: jpa-00249277 https://hal.science/jpa-00249277}

Submitted on 1 Jan 1994

HAL is a multi-disciplinary open access archive for the deposit and dissemination of scientific research documents, whether they are published or not. The documents may come from teaching and research institutions in France or abroad, or from public or private research centers.
L'archive ouverte pluridisciplinaire HAL, est destinée au dépôt et à la diffusion de documents scientifiques de niveau recherche, publiés ou non, émanant des établissements d'enseignement et de recherche français ou étrangers, des laboratoires publics ou privés. 
Classification

Physics Abstracts

42.65

\title{
Weakly multimode dynamics of a photorefractive oscillator
}

\author{
D. Hennequin, L. Dambly, D. Dangoisse and P. Glorieux \\ Laboratoire de Spectroscopie Hertzienne, Unité associée au Centre National de la Recherche \\ Scientifique, Université des Sciences et Technologies de Lille, 59655 Villeneuve d'Ascq Cedex, \\ France
}

(Received 27 January 1994, accepted 2 May 1994)

\begin{abstract}
The dynamics of a photorefractive oscillator has been studied in the weakly multimode case. It is shown that the eigenmodes of the empty cavity provide the bases in which the transverse patterns of this oscillator should be analysed. The concept of mode families i.e. sets of frequency degenerate modes, is very efficient to classify the different ranges of operation of the oscillator with Fresnel number up to 5-7. As the complexity of the patterns increases, the interpretation in terms of optical defects or phase singularities greatly simplifies the analysis.
\end{abstract}

Photorefractive materials have been shown to be efficient in many applications, such as pattern recognition, volume holographic storage, phase conjugated lasers, . [1]. The knowledge and the understanding of the dynamics that they could develop when they are placed inside a cavity is important from two points of view : (i) they can help in building devices whose stable patterns are used as a basis for the manipulation of complex images, for example by reducing images to the components of their projection on a limited number of « eigen-patterns » [2] ; (ii) more fundamentally, photorefractive oscillators (PRO) have analogies with lasers [3], and thus could contribute to understand the spatio-temporal dynamics of nonlinear optical systems. Indeed, some properties of PROs, and in particular their relatively long response time, allow us to access more easily to the information needed to analyse the spatio-temporal behavior of nonlinear optical systems. In fact, as the analogy with lasers has been demonstrated through the Ginzburg-Landau equation, dynamics of photorefractive oscillators should show analogies with a much larger class of physical systems, namely those in which complex spatio-temporal behaviors lead to turbulence.

In order to determine the basic mechanisms of transverse dynamics in nonlinear optical systems, we have chosen to study first the behavior of a simple system with few spatiotemporal degrees of freedom. Here we have used an optical cavity whose gain is provided by two-wave mixing ( $2 \mathrm{WM}$ ) in a photorefractive crystal, and with small transverse dimensions such that only few modes are able to oscillate in the empty cavity. By varying the diameter of an intracavity aperture, we change the number of degrees of freedom of the transverse variables and study the evolution of transverse patterns in situations of different complexity. The dynamics is interpreted in terms of the empty-cavity modes (ec modes) and of their 
interactions. The paper is organized as follows : we present first the experimental set-up and recall the particular properties of the photorefractive crystals relevant to this work, together with some notations concerning optical cavities. The next section deals with the global response of the oscillator as a function of the gain and the cavity length. We show in particular that in spite of fundamental differences between PROs and lasers, the ec modes play a similar role in both systems. Parts 3 and 4 describe respectively the stationary and dynamical patterns involved by the PRO, and interpretate them through the interactions between ec modes. In part 5, we briefly discuss the results and their relevance to the future investigation of systems of higher complexity.

\section{Experimental set-up.}

The experimental set-up is shown in figure 1 . The cavity is limited by three plane mirrors $\mathbf{M}$ and a beam splitter BS coupling $10 \%$ of the beam outside the cavity. One of the mirrors is mounted on a PZT translator in order to vary the total length $L$ of the cavity at the scale of the optical wavelength $\lambda$. A focusing lens with focal length $f$ is added to stabilize the cavity. An iris allows us to restrict the transverse spatial extension of the beam inside the cavity. The gain of the oscillator is obtained by $2 \mathrm{WM}$ inside a $\mathrm{Bi}_{12} \mathrm{SiO}_{20}$ (BSO) photorefractive crystal. The crystal is pumped with a frequency doubled YAG laser $(\lambda=532 \mathrm{~nm}$ ) with an intensity of about $10 \mathrm{~mW} / \mathrm{cm}^{2}$. The angle between the pump beam and the cavity axis is about $3^{\circ}$. The light emitted by the PRO is monitored by a CCD camera which is connected to a Video Cassette Recorder to record long time sequences and to a computer to analyse the instantaneous patterns. Heterodyne detection can also be performed by mixing the signal with the pump beam.

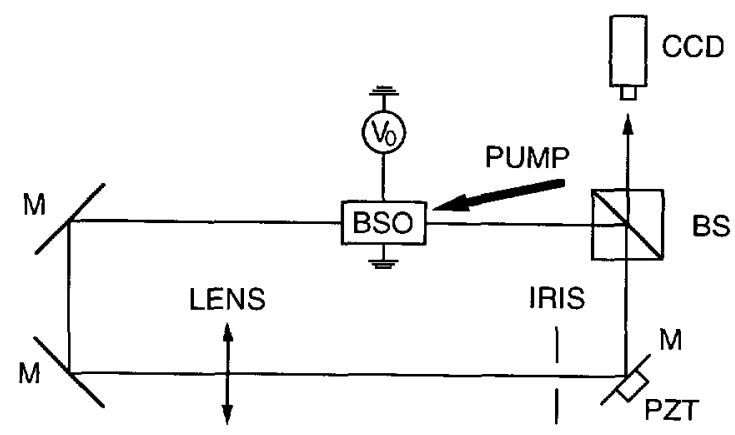

Fig. 1. - Experimental set-up of the 2 WM PRO. The cavity is limited by three flat mirrors $\mathrm{M}$ and a beam splitter BS. The lens stabilizes the cavity. The piezoelectric translator PZT permits the cavity length to be varied.

When both a probe and a pump radiation with frequencies $\nu_{\mathrm{r}}$ and $\nu_{\mathrm{p}}$ respectively are sent onto a photorefractive crystal adequately oriented, a part of the pump energy is transferred to the probe beam. The transfer efficiency depends on the angle between the two beams and their frequency difference. It increases with the ratio of the pump to the probe intensities. In a cavity such as that of figure 1, when the probe power is initially zero, the oscillation is triggered by the noise falling inside the gain bandwidth of the crystal. The gain curve is centered on frequency $\nu_{0}$, detuned from $\nu_{\mathrm{p}}$ by $6 \mathrm{~Hz}$ typically. This triggering optical noise originates essentially from the diffusion of the pump radiation inside the crystal. Here the value of the gain is linked to the motion of electric charges inside the crystal. In BSO, this gain is zero 
except if a DC electric field $E_{0}$ is applied to the crystal. Therefore, this parameter controls the values of the round trip gain; when the other parameters are optimized, the variation of $E_{0}$ from 0 to $10 \mathrm{kV} / \mathrm{cm}$ induces a variation of the gain from 0 to $1000 \%$ typically. This high gain allows us to use a «bad» cavity, which is effectively the case owing to the large losses introduced by the crystal itself (transmission and reflection losses). The total losses per round trip are estimated to be $75 \%$, leading to a small cavity finesse of 2.1 .

The theory of empty cavities shows that the longitudinal and transverse boundary conditions lead to Gaussian eigenmodes belonging to bases such as the Hermite-Gauss or the LaguerreGauss ones. The Laguerre-Gauss modes, corresponding to a cylindrical transverse symmetry, as that imposed by the circular iris in our set-up, will be denoted in the following $\mathrm{A}_{p \ell_{1}}$, where $p$ is the radial index, $\ell$ the longitudinal one and $i=1$ (resp. 2) for a cosine (resp. sine) mode [4]. The total length of the cavity and the focal length of the lens determine the stability of the cavity, and thus the arrangement of the mode frequencies. The free spectral range of the cavity, i.e. the spacing between two successive longitudinal modes, is $\Delta \nu_{\mathrm{L}}=c / L$. The frequency detuning of the $\mathrm{A}_{p \ell_{1}}$ transverse mode with respect to the fundamental $\mathrm{A}_{00}$ mode is

$$
\Delta \nu_{p \ell}=(2 p+\ell) \frac{\Delta \nu_{\mathrm{L}}}{\pi} \cos ^{-1} \sqrt{1-\frac{L}{4 f}}=(2 p+\ell) \Delta \nu_{\mathrm{T}} .
$$

Therefore, once all the cavity parameters are fixed, the frequency of a transverse mode is given by the index $\xi=2 p+\ell$, and modes with same $\xi$ have same frequency. Such sets of frequency-degenerate modes are called «mode families» [4]. In fact, modes of the same family are frequency degenerated only in the "ideal» case. Practically, breaking of the cylindrical symmetry of the cavity, by e.g. misalignment or astigmatism, lifts this frequency degeneracy. Thus the length of the cavity, the focal length of the lens and the global symmetry of the cavity are important geometrical factors which have been chosen according to the needs of each series of experiments. Parameters such as the transverse and longitudinal intermode spacings or the effective symmetry of the cavity are adjusted by acting on these quantities. Typical values are $f=0.5 \mathrm{~m}$ and $L$ between 1 and $3 \mathrm{~m}$, leading to intermode spacings in the range of several tens of $\mathrm{MHz}$.

The transverse spatial extension is a key quantity of our experiments. As the laser electromagnetic field energy is confined in a region whose diameter increases for higher order transverse modes, this transverse extension governs the number of transverse modes able to be transmitted by the empty cavity. This quantity may be measured by the generalized Fresnel number $\mathcal{N}$ :

$$
\mathcal{N}=\left(\frac{\Sigma}{\pi u^{2}}\right)_{\min }
$$

where $w$ is the spot size of the fundamental mode and $\Sigma$ is the cross section of the cavity [5]. The index indicates that the Fresnel number corresponds to the smallest value of this quantity as the field travels along the cavity. In our case. the Fresnel number must be calculated in the plane of the iris, and so it is equal to

$$
r=\left(a / u^{\prime}\right)^{2}
$$

where $a$ is the radius of the iris aperture. The results presented in the following correspond to typical $\mathcal{N}^{3}$ values ranging from 1 to 10 . 


\section{Phase diagram of the PRO.}

Although we have not made any quantitative check of this point, it immediately appears that the patterns emitted by the active cavity are extremely similar to ec modes, in particular Laguerre-Gauss modes and we will use the labeling of these modes to describe them. Figure 2 shows the phase diagram of the PRO as a function of both the cavity length and the external DC field for a cavity with $\Delta \nu_{\mathrm{T}}=67 \mathrm{MHz}, \Delta \nu_{\mathrm{L}} / \Delta \nu_{\mathrm{T}}=2.5$ and $\mathcal{N}=5$. In this diagram, the numbers indicate the mode family which is oscillating in the considered area. Hatched areas correspond to non oscillating regions. The behavior described by this diagram is very similar to that observed in lasers. For a given length of the cavity, the PRO oscillates on the nearest ec modes. To overcome the losses, a minimum gain is required for oscillation, determining a threshold as in a laser. As the losses induced by the iris increase with $\xi$, the threshold is generally lower for small $\xi$ mode families.

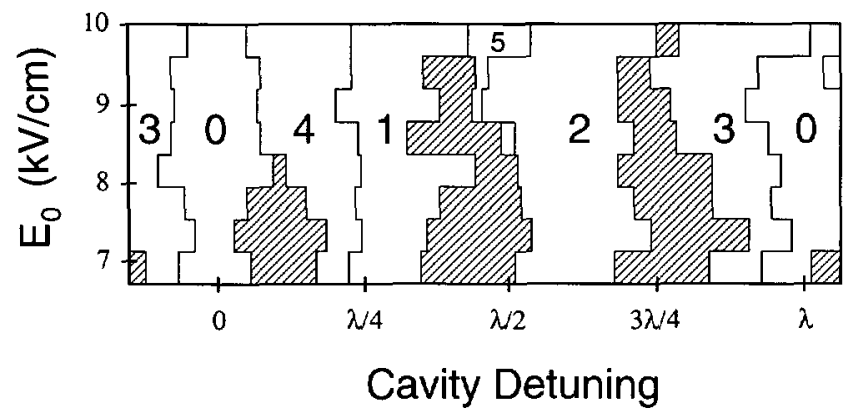

Fig. 2. - Phase diagram of the PRO as the cavity detuning and the crystal voltage $V_{0}$ are varied. The cavity detuning is given in length units, and $E_{0}$ is the electric field associated with $V_{0}$. The regions in which the PRO do not oscillate are hatched. In the other areas, a number indicates the family that oscillates.

This first result is not so trivial in the particular situation of the PRO, as compared with the usual lasers. In the latter, the gain curve is much larger than the empty cavity linewidth, which is directly linked to the losses of the cavity for the considered mode. As a consequence, the emission frequency is always relatively close to the frequency of the corresponding ec mode. On the opposite, the gain curve in the PRO is very narrow, about $10 \mathrm{~Hz}$ in our case, and so the emission frequency of the PRO remains practically unchanged when the cavity length is scanned. The fact that the PRO oscillates for almost any cavity length when the gain is large enough. indicates that a very strong frequency pulling exists, as it is predicted by theory [6]. Moreover, the strong frequency pulling is favoured here by the fact that due to large cavity losses, the ec modes linewidth is also large.

This interpretation is confirmed by the measure of the output frequency of the PRO as the cavity length is swept. This can be done easily through an heterodyne detection of the emitted field. When mixing the output beam of the PRO with that of the pump, the pattern exhibits fringes moving with a speed directly related to the difference $\Delta \nu$ between the two beam frequencies. As the cavity length is increased in the range of existence of a given pattern, i.e. typically $\lambda / 5$, corresponding to a shift of the ec mode frequency of $67 \mathrm{MHz}$ in the conditions of figure $2, \Delta \nu$ varies typically from 4 to $8 \mathrm{~Hz}$. These values are independent of the considered mode. 
The phase diagram also indicates that the oscillating regions of each family are well delimited, and that no overlapping of families was observed. This corresponds to the fact that all the observed patterns are always combinations of mode of the same family, and that when a parameter is changed so that the border between two families is crossed, the PRO jumps abruptly from a pattern described by modes of the first family to a pattern described by modes of the second family.

In fact, the situation described by figure 2 is very simple, as the configuration of the cavity and the Fresnel number have been chosen so that the ec modes involved in the PRO dynamics are grouped in families separated by several tens of $\mathrm{MHz}$. For other cavity configurations or larger Fresnel numbers, it often happens that two families of different longitudinal index are very close, sometimes separated only by a few $\mathrm{kHz}$ or less. If this difference is small enough, typically in the range below $1 \mathrm{MHz}$, modes of both families participate to the dynamics. This point will be discussed with more details in the next sections.

\section{Stationary patterns of the PRO.}

As mentioned above, when the Fresnel number remains small i.e. of the order of 3 to 5 typically, the observed patterns can always be easily interpreted as linear combinations of few ec modes. Almost all combinations could be observed, depending on the geometry, the gain and the transverse symmetry of the cavity, and more generally on the ec modes whose frequency falls near the pump frequency.

When the situation is such that only one isolated mode has a resonance frequency close to $\nu_{0}$, the output pattern of the PRO looks very similar to that of this mode. Figure 3 shows examples of the modes $A_{00}, A_{011}, A_{022}$ and $A_{031}$, belonging respectively to the $\xi=0,1,2$ and 3 families, together with their theoretical shape. This list is not exhaustive, as theoretically all ec modes should be observable. In practice, it is true only for small $\xi$-modes. Indeed, as $\xi$ increases. it is more and more difficult to separate mode frequencies, as many other modes are able to oscillate and come to interfere with the considered ones. Let's take an example : to
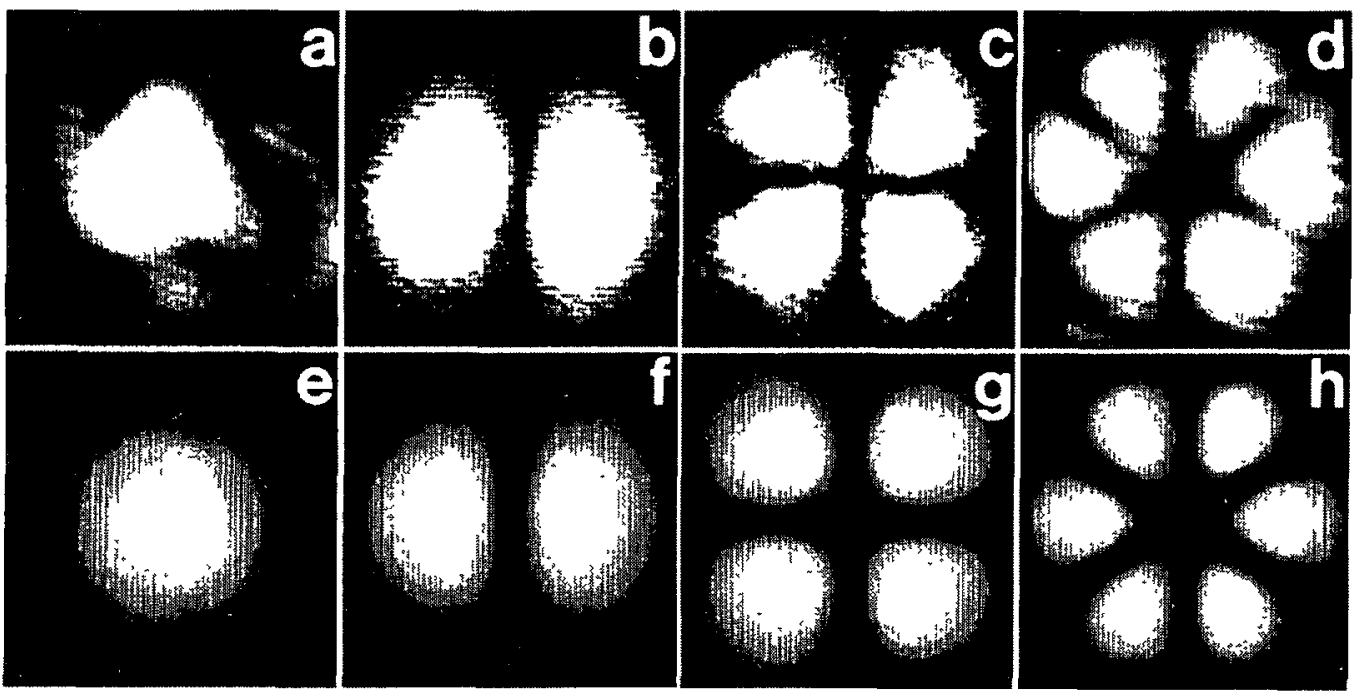

Fig. 3. - Intensity distribution of some monomode stationary observed patterns : (a) $A_{00}$ mode ; (b) $A_{011}$ mode ; (c) $A_{022}$ mode ; (d) $A_{031}$ mode. Patterns (e), (f). (g) and (h) represent the theoretical shapes of $A_{00}, A_{011}, A_{022}$ and $A_{031}$ modes respectively. 
observe the $A_{011}$ mode, it is necessary to lift the degeneracy between this mode and the $\mathrm{A}_{012}$ mode. In practice, it is easy to choose a cavity configuration such that the $\xi=0$ and $\xi=1$ families are well separated, say $\Delta \nu_{\mathrm{T}} / \Delta \nu_{\mathrm{L}}=2$, and to reduce the iris aperture so that families with $\xi>1$ cannot oscillate. Then it remains to introduce enough astigmatism to separate the two $\xi=1$ modes. As the length of the cavity is scanned, three well separated modes appear: $\mathrm{A}_{00}, \mathrm{~A}_{011}$ and $\mathrm{A}_{012}$. The observation of a higher $\xi$-mode, as e.g. the $\mathrm{A}_{041}$ mode, is much more difficult. It is necessary to choose $\Delta \nu_{\mathrm{T}} / \Delta \nu_{\mathrm{L}}=5$, and then to separate the five modes of the $\xi=4$ family. As the cavity length is scanned, up to 15 modes appear successively, with a frequency spacing smaller than in the previous case. This sets a practical limit to the maximum order of the family that can be observed in stationary operation.

When the frequency spacing between two transverse modes is small enough, the observed stationary pattern is a linear combination of these two modes. More generally, when the Fresnel number, and so the mode-spectrum density, is increased, the stationary patterns are often a combination of several ec modes. For example, in the $\xi=1$ family, when the two modes $A_{011}$ and $A_{012}$ have the same frequency, the observed pattern is no longer one of these two modes, but a linear combination of them as shown in figures $4 \mathrm{a}$ and $6 \mathrm{a}$. These two patterns have an intensity distribution corresponding to a field distribution of the type

$$
A_{1} \mathrm{e}^{t \omega t}+A_{2} \mathrm{e}^{t(\omega t+\varphi)}
$$

where $A_{1}$ and $A_{2}$ are the field amplitudes of the $\mathrm{A}_{011}$ and $\mathrm{A}_{012}$ modes and $\omega$ is the light frequency. Figure 6 a (resp. fig. 4a) corresponds to $\varphi=\pi$ (resp. $\varphi=\pi / 2$ ) [7]. As in typical coupled oscillators, the single frequency $\omega$ that appears in equation (1) could result from locking of slightly different ec mode frequencies [8]. We have qualitatively observed that this effect in the PRO happens for very small frequency differences, smaller than the precision of the cavity length adjustment, i.e. typically $10 \mathrm{kHz}$.

Patterns of figures $4 a$ and $6 a$ are a good illustration for another approach of the characterization of patterns that seems to be more suitable in most cases. Indeed, the pattern of figure 6a appears to be equivalent to the $\mathrm{A}_{01}$ mode with a change in the reference axes, owing e.g. to a small breaking of the transverse symmetry of the cavity. In the same way, the pattern of figure $4 \mathrm{a}$ is obtained in a cavity with cylindrical symmetry. without any preferred axis, and so is a sum of the two ec modes of the family. The particular phase of $\pi / 2$ seems to be typical of the interaction of modes inside one family, as we will see later.

The pattern of figure $4 \mathrm{a}$ exhibits in its center a point with special properties. This point of zero intensity, and thus of zero field amplitude, is a phase singularity, i.e. a point where the
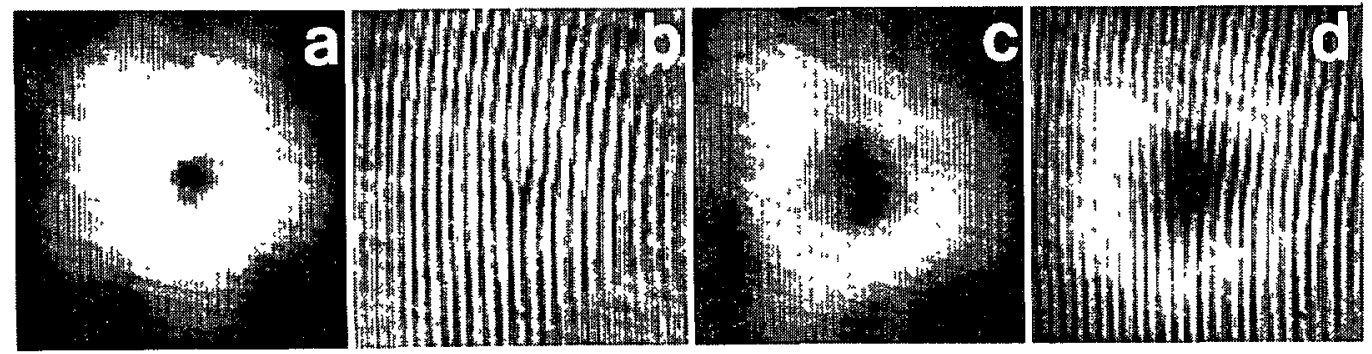

Fig. 4. - Experimental recording of the $\xi=1$ and $\xi=2$ doughnuts. (a) intensity distribution of the $\xi=1$ doughnut; (b) heterodyne detection of (a) showing a phase singularity with charge $=+1$ in the center ; (c) intensity distribution of the $\xi=2$ doughnut; (d) heterodyne detection of (b) showing a phase singularity with charge $=+2$ in the center. In (a) and (c), fringes result from technical noise. 
phase is not defined and cannot be defined by continuity [9]. This appears clearly when an heterodyne detection of this pattern is used (Fig. 4b). A defect similar to a dislocation appears in the fringe grating: one fringe splits suddenly into two at this point, and therefore, the circulation of the phase around this point is nonzero, but equal to $m 2 \pi$, where $m$ is a positive or negative integer, and is called the "topological charge » of the phase singularity. Such phase singularities, also called « optical vortices», should play an important role in the highly multimode dynamics of PROs, nonlinear passive Fabry-Pérot interferometers [10] and lasers, and in particular could be at the origin of optical turbulence [9]. Therefore it is interesting to consider what role they play in weakly multimode dynamics. Moreover, they introduce a new tool in understanding patterns, through the charges of their phase singularities. Experimentally, the vortex charge can be easily determined: when turning around the singularity, the charge is the difference between the number of fringes crossed in the top halfcircle and that in the bottom half-circle. The vortex in figures $4 a, b$ appears thus to have a charge of +1 . Figure $4 c$ shows a pattern that resembles very much that of figure $4 a$. However, its heterodyne counterpart reveals that its central vortex has a charge of +2 . In fact, the doughnut of figures $4 \mathrm{c}$, d belongs to the $\xi=2$ family : only the examination of the charge of the phase singularity allows us to differentiate it from that of figures $4 a, b$.

Figures 5 and 6 shows some other examples of stationary patterns. As above, different points of view may be adopted. Patterns may be considered as combinations of two or more ec modes. Thus, pattern 5a is a combination of the three ec modes of the $\xi=2$ family, pattern $5 \mathrm{c}$ is a
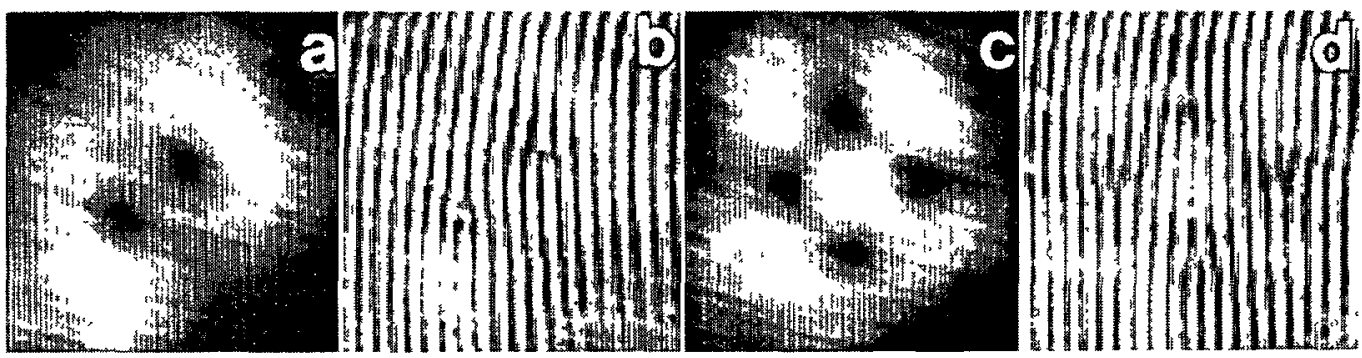

Fig. 5. - Experimental recording of $\xi=2$ patterns. (a) intensity distribution of a two-vortex pattern : (b) heterodyne detection of (a) : the two vortices have charge $=-1$; (c) intensity distribution of a fourvortex pattern; (d) heterodyne detection of (b) : the vortices have charges $= \pm 1$. In (a) and (c), fringes result from technical noise.
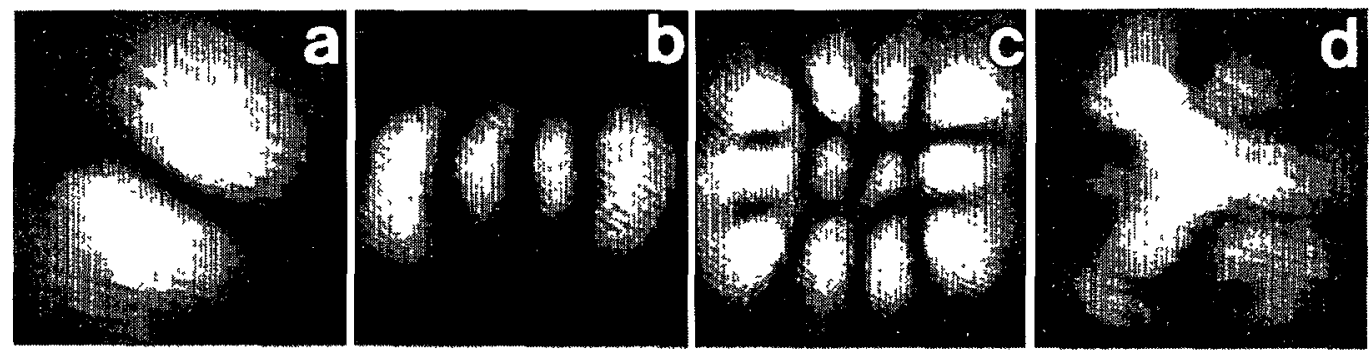

Fig. 6. - Intensity distribution of some multımode stationary patterns: (a) $\xi=1$; (b) $\xi=3$; (c) $\xi=5 ;$ (d) combination of $\xi=0$ and $\xi=3$. 
combination of $A_{10}$ and $A_{022}$, patterns $6 \mathrm{~b}$ and $6 \mathrm{c}$ may be found back from ec modes of the $\xi=3$ and $\xi=5$ families respectively, while finally pattern $6 \mathrm{~d}$ is a combination of $A_{031}$ and $A_{00}$. When the situation is simple, as e.g. for patterns of figures $5 \mathrm{c}$ and $6 \mathrm{~d}$, we observed that the phase that appears in the two-mode combination is different depending on whether these two modes belong to the same family or not. The pattern of figure $5 \mathrm{c}$ is solution of equation (1) for the $A_{10}$ and $A_{022}$ modes and $\varphi=\pi / 2$, while the pattern of figure $6 \mathrm{~d}$ corresponds to $\mathrm{A}_{00}$ and $\mathrm{A}_{031}$ modes with $\varphi=0$. In the first case, the two modes belong to the same family and they add in quadrature, while in the second case, the two modes originate from different families and add in phase. This behavior seems to be general, at least for twomode combination, and when the symmetry of the system is taken into account, choosing for example appropriate axes to build the ec modes.

Indeed, this approach using ec modes becomes difficult to use when more than three modes are interacting. However, if symmetries of the pattern are taken into account, this approach may be extended through the use of ec modes of other Gaussian bases. In this case, patterns such as those of figures $6 \mathrm{~b}$ or $6 \mathrm{c}$ may be easily identified, since they are eigenmodes of the Hermite-Gauss basis, respectively the $\mathrm{TEM}_{30}$ and $\mathrm{TEM}_{32}$ modes. This type of solution results probably from a change of the transverse symmetry of the cavity, originating in the adjustments of the system to obtain these patterns, and making the symmetry closer to the rectangular one.

However, the previous method of characterization of the patterns appears to be inadequate as the number of modes increases or when temporal dynamics is considered. Let us explain first how phase singularities can help in simplifying the analysis of data on transverse dynamics. Stationary patterns with respectively 2 and 4 vortices are shown in figures $5 \mathrm{a}$ and $5 \mathrm{c}$ with their corresponding heterodyne detections in $5 \mathrm{~b}$ and $5 \mathrm{~d}$. Patterns with up to 7 vortices have currently been observed. Different distributions of charges are found. In figures $5 \mathrm{a}, \mathrm{b}$, both phase singularities have a charge -1 , while in figures $4 c, d$, two of charge +1 coexist with two of charge -1 . These vortices are always ordered following the rule which tells that when their charges have the same sign, they repel each other, while they attract each other if they have different signs [4]. In patterns where vortices have all the same charge, as in figure 5a (see also Fig. 9 for three vortices of charge -1 ), they are regularly spaced. When opposite charges coexist, stationarity is obtained through the formation of crystals where phase singularities of opposite charges alternate. This type of geometry for charges of different signs have been theoretically predicted through an analogy with electric charges of particules, and was already observed in lasers $[4,11]$.

\section{Dynamical patterns of the PRO.}

In the previous sections, two types of interactions between ec modes have been described depending on the frequency difference between these modes: when ec modes are well separated, mutual exclusion arises so that the PRO oscillates only on the mode with the frequency closest to the central gain frequency; when ec modes have almost the same frequency, stationary patterns composed of a mixture of these particular modes are observed. In the scale of the frequency spacing between modes, there exists an intermediate situation where, although the modes have well different frequencies, they participate together to the PRO operation. In this case, corresponding typically to spacing of $1 \mathrm{MHz}$ and less, periodic regimes have been observed. Let us remember that frequency locking, as discussed in section 3, appears for at least two-order-of-magnitude-smaller frequency spacing.

Figures 7,8 and 9 show sequences recorded from typical periodic regimes. The four patterns are snapshots separated by $0.5 \mathrm{~s}$ (Fig. 7), $0.24 \mathrm{~s}$ (Fig. 8) and $0.28 \mathrm{~s}$ (Fig. 9), and represent the evolution of the output pattern of the PRO during half a period (Figs. 7 and 8 ) or one third of a 

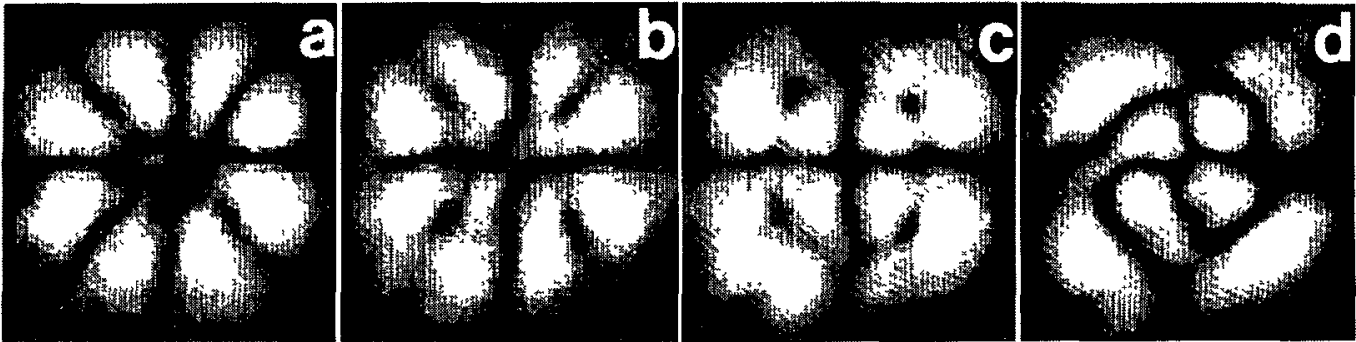

Fig. 7. - Half a period of a $\xi=4$ periodic pattern. Snapshots are separated by $0.5 \mathrm{~s}$. The second half of the period is similar to this sequence, but reversed in time.
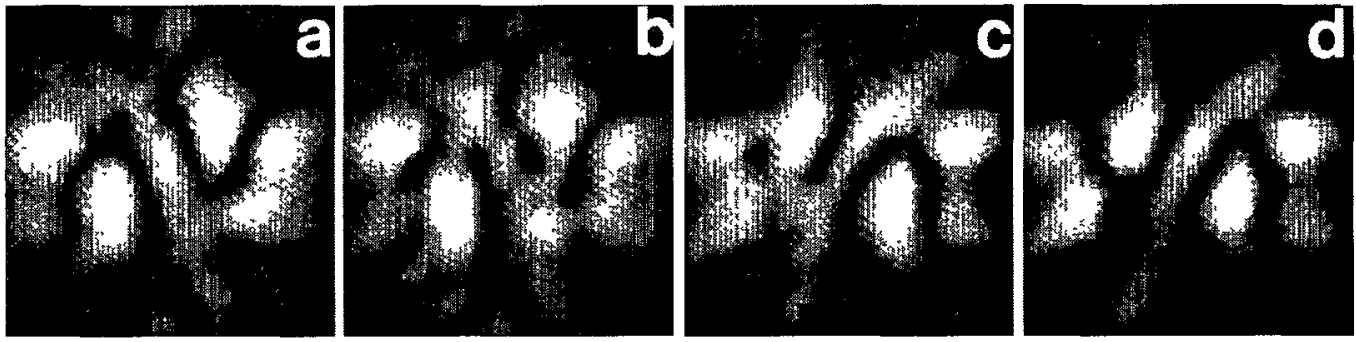

Fig. 8. - Half a period of a periodic pattern combining the $\xi=1$ and $\xi=4$ families. Snapshots are separated by $0.24 \mathrm{~s}$. The second half of the period is similar to this sequence, but reversed in time.
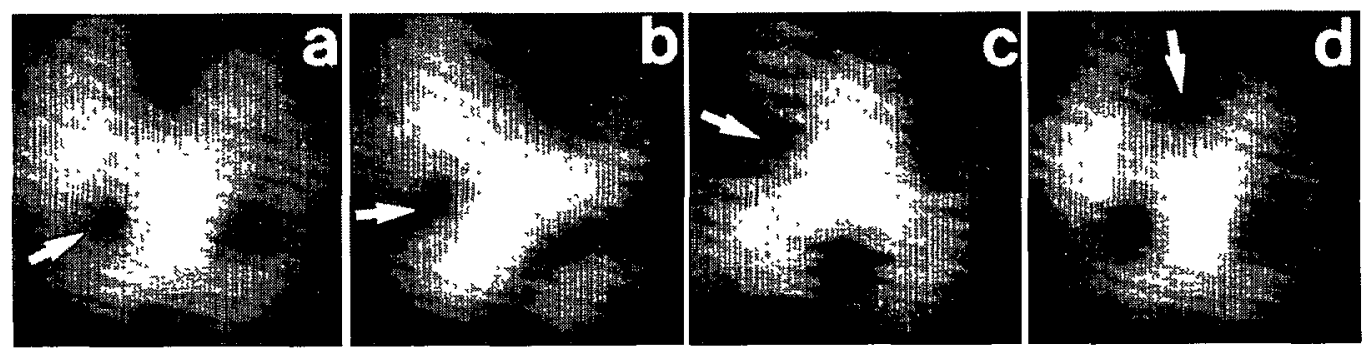

Fig. 9. - One third of the period of a periodic pattern combining the $\xi=0$ and $\xi=3$ families. Snapshots are separated by $0.28 \mathrm{~s}$. The dynamics of the pattern consists in the rotation of three equidistant vortices around the pattern center. For sake of clarity, one of the vortices has been marked by a white index.

period (Fig. 9). The period $\Omega^{-1}$ is $3 \mathrm{~s}, 1.6 \mathrm{~s}$ and $2.6 \mathrm{~s}$ for figures 7,8 and 9 respectively. In figures 7 and 8 , the second half of the period follows the same evolution as that shown, but reversed in time. In figure 9 , the dynamics consists in a continuous rotation of the pattern around its center. Equation (1) is no longer suitable to describe this behavior, as a second frequency, much lower than the optical one, has appeared. The introduction of a small frequency in equation (1) leads to the following expression :

$$
A_{1} \mathrm{e}^{l \Omega t} \mathrm{e}^{l \omega t}+A_{2} \mathrm{e}^{t(\Omega t+\phi)} \mathrm{e}^{t(\omega t+\varphi)}
$$


where $\omega$ and $\Omega$ are linked to respectively the half sum and the half difference of the frequencies of modes $A_{1}$ and $A_{2}$ [7]. The fact that very low $\Omega$ frequencies are observed in the $\mathrm{PRO}$ in the range of one $\mathrm{Hz}$, although interacting ec modes may be separated by $\mathrm{MHz}$, is not so surprising. Indeed, as already discussed in section 2, ec mode frequencies in the PRO are shifted by a very strong frequency pulling which squeezes all the active optical frequencies inside the gain curve, wide of only a few $\mathrm{Hz}$ [6]. As a consequence, the differences between active optical frequencies are also of the order of one $\mathrm{Hz}$.

Using expression (2), the pattern shown in figure 7 appears as resulting from the interaction of the two modes $\mathrm{A}_{042}$ and $\mathrm{A}_{112}$, both belonging to the $\xi=4$ family, with phases $\phi=\pi / 2$ and $\varphi=\pi / 2$. As for stationary patterns, the interaction of modes belonging to the same family is associated with $\varphi=\pi / 2$. In the same way, the pattern of figure 8 appears as the interaction of $\mathrm{A}_{012}$ with modes of the $\xi=4$ family combined to form the $\mathrm{TEM}_{40}$ HermiteGauss mode. The phases in equation (2) are here $\phi=\pi / 2$ and $\varphi=0$. When symmetries are taken into consideration, this rule seems to be general : modes belonging to the same family interact through a $\pi / 2$ phase, while modes from different families interact through a zero phase.

Figure 9 gives another illustration of a dynamical pattern resulting from the interaction of modes belonging to two different families, namely the $\xi=0$ and $\xi=3$ ones. The $\xi=3$ mode appearing here as a doughnut mode, is the superposition of the two $\mathrm{A}_{03}$ modes. However, the approach from the point of view of phase singularities is more appropriate : the dynamics consists in the rotation of three vortices of charge -1 around the center of the pattern. The three vortices are always equidistant, following their electrie-chargelike behavior. Such transverse travelling waves corresponding to the rotation of phase singularities have already been predicted and observed in lasers [11]. Depending on the sign of the charges, they can be associated with creation and annihilation of vortices. In the case of figure 9 , the three vortices have the same charge, and so annihilation is not possible, as all vortices repel each other. The dynamics may be more complex : for example, when all three modes of the $\xi=2$ family compete, the vortices exhibit a complex motion including rotation, precession, and cycles of annihilation-creation, as those observed in lasers [7, 11]. These are just preliminary examples, and the dynamics resulting from the interaction of a great number of vortices, is now being considered.

\section{Conclusion.}

We have reported here the simple basic dynamics of PROs in the case of weakly multimode cavities. The observed behaviors are either stationary or time periodic and the patterns are often very similar to the empty cavity modes. The analysis and the understanding of the dynamics using mode properties have been done taking into account the symmetry of the cavity. It results from this approach that the behavior of the PRO is very sensitive to different parameters as the symmetry of the cavity or the frequency spacing between modes, and that the adjustment of these parameters leads to a very rich phenomenology. In future investigations, this high sensitivity could be used in pattern recognition devices taking advantages of the spontaneous symmetry breakings of the behavior [12].

The PRO provides also a good system to study complex spatio-temporal dynamics and optical turbulence. This paper shows that even in simple behaviors, consideration of phase singularities as «charged pseudo-particles» appears crucial to extract simplified information on the observed patterns, and the temporal evolution of the patterns is interpreted in terms of the motion of these « charged pseudo-particles ». By increasing the aperture of the intracavity diaphragm, it is possible to explore a region of larger Fresnel numbers where more modes should interact and lead to larger complexity. The statistical study of phase singularities has 
already be done [13] but the dynamics of these complex systems still remains to be explored. Owing to their long time scale as compared with usual lasers, PRO's appear as a good system to perform such studies.

The PRO's, as the lasers, are the association of an optical self-amplifier with a cavity, and thus it is interesting to compare their properties. The main differences result from the nature of the PRO gain process, $2 \mathrm{WM}$ instead of stimulated emission, and from its very narrow bandwidth. Therefore, the emission frequency of the PRO varies only on a few Hertz, which is possible only because of a huge frequency pulling. However, in spite of these strong differences, we have shown that the behavior of the PRO is very similar to that of lasers. In particular, the cavity parameters, such as the resonator length or detuning, appear to play the same selecting role as in lasers. Moreover, most of the weakly multimode stationary and dynamical patterns described in this paper have been observed or predicted in laser systems [4, 11]. Some simple models already propose an approach to explain these similarities, through a common description in the near-threshold region by the Ginzburg-Landau equation [3]. Therefore, the results obtained in the PRO's could be extended not only to the lasers, but also to a larger class of systems modelized by this equation.

\section{References}

[1] Rajbenbach H., Bann S. and Huignard J.-P., Long-term readout of photorefractive memories by using a storage/amplification two-crystal configuration, Opt. Lett. 17 (1992) 1712-1714. See also references herein.

[2] Brambilla M., Lugiato L. A., Pinna M. V., Prati F., Pagani P., Vanotti P.. Li M. Y. and Weiss C. O., The laser as nonlinear element for an optical associative memory, Opt. Commun. 92 (1992) 145.

[3] Tarroja M. F. H., Staliunias K., Slekys G., Weiss C. O. and Dambly L., Analogy between photorefractive oscillators and class-A lasers, submitted to Phys. Rev A.

[4] Brambilla M., Battipede F., Lugiato L. A., Penna V., Prati F., Tamm C. and Weiss C. O., Transverse laser patterns, Phys. Rev. A 43 (1991) 5090-5120.

[5] Dangorsse D., Hennequin D., Lepers C., Louvergneaux E. and Glorieux P., 2-D optical lattices in a $\mathrm{CO}_{2}$ laser, Phys. Rev. A 46 (1992) 5955-5958.

[6] Dambly L. and Zeghlache H., Frequency shift in an oscillator with photorefractive gain, Phys. Rev. A 47 (1993) 2264-2275.

[7] Hennequin D., Dambly L., Dangoisse D. and Glorieux P., Basic transverse dynamics of a photorefractive oscillator, $J$ Opt Soc. Am. B 11 (1994) 676-684

[8] Adler R., Proc. I R.E. 34 (1946) 351.

[9] Coullet P., Gil L. and Rocca F., Optical vortices, Opt. Commun. 73 (1989) 403-408;

Coullet P., Gil L. and Lega J., Defect mediated turbulence, Phys. Rev. Lett. 62 (1989) 1619-1622.

[10] Neubecker R., Kreuzer M. and Tschudi T., Phase defects in a nonlinear Fabry-Pérot resonator, Opt. Commun 96 (1993) 117-122.

[11] Brambilla M., Cattaneo M. L., Lugiato L. A.. Prrovano R., Prati F., Kent A. J., Oppo G. L., Coates A. B., Weiss C. O., Green C., D'Angelo E. J. and Tredicce J. R., Dynamical transverse laser patterns I, Phys. Rev. A 49 (1994) 1427-1451.

Coates A. B.. Weiss C. O., Green C., D'Angelo E. J., Tredicce J. R., Brambilla M., Cattaneo M., Lugiato L. A., Pirovano R., Prati F., Kent A. J. and Oppo G. L., Dynamical transverse pattern II, Phys Rev A 49 (1994) 1452-1466.

[12] Smith C. P., Dihardja Y., Weıss C. O., Lugıato L. A., Prati F. and Vanottı P., Low energy switching of laser doughnut modes and pattern recognition, Opt Commun. 102 (1993) 505-514.

[13] Arecchi F. T., Giacomelli G., Ramazza P. L. and Residori S., Vortices and defect statistics in twodimensional optical chaos. Phys. Rev. Lett. 67 (1991) 3749-3752. 